\title{
The Role of Serum Cytokeratin 19 Fragment in Transarterial Infusion against Invasive Bladder Cancer
}

\author{
Ryo Takahashi", Ryoji Kimata, Shunichiro Nomura, Ichiro Matsuzawa, Yasutomo Suzuki, \\ Tsutomu Hamasaki, Yukihiro Kondo \\ Department of Urology, Nippon Medical School, Tokyo, Japan \\ Email:"s7053@nms.ac.jp
}

Received May 6, 2013; revised June 4, 2013; accepted June 11, 2013

Copyright (C) 2013 Ryo Takahashi et al. This is an open access article distributed under the Creative Commons Attribution License, which permits unrestricted use, distribution, and reproduction in any medium, provided the original work is properly cited.

\begin{abstract}
Background: We used transcatheter arterial infusion chemotherapy (TAI) for patients with T1G3 and greater than T2 bladder cancer, which was diagnosed after extensive and deep transurethral resection of bladder tumor (TUR-BT), and we investigated the utility of serum cytokeratin 19 fragment (CYFRA) as a predictive factor of the response to therapy. Material and Methods: From November 2001 to November 2010, 56 patients (46 males and 10 females) with pathologically confirmed T1 G3 or greater than T2 bladder cancer after TUR-BT underwent two courses of TAI of cisplatin, methotrexate and doxorubicin as neoadjuvant setting. Then, patients underwent evaluation TUR-BT. Thereafter, the bladder was preserved in patients with superficial or undetectable tumors on TUR-BT. Advanced cases and residual bladder tumor cases were treated with total cystectomy or systemic chemotherapy. CYFRA levels were measured before and after performing TAI. Results: With this therapy, the 5 -year survival rate was $85.7 \%$ in pT1G3, $82.3 \%$ in pT2, and $66.6 \%$ in greater than pT3 cases. Bladder preservation with no recurrence was observed in $58.7 \%$ of the patients. Grade III adverse events included leucopenia (6/56 patients: 10.7\%). Serum CYFRA levels significantly decreased with treatment (in 4/6 patients with elevated CYFRA levels). Conclusion: These results suggest that although total cystictomy is usually indicated for T1G3 bladder cancers, bladder preservation and control of micrometastases can be achieved by performing TAI after extensive and deep TUR-BT. Our results also suggest the utility of monitoring serum CYFRA to assess the response to therapy.
\end{abstract}

Keywords: Transcatheter Arterial Infusion (TAI); Invasive Bladder Cancer; Cytokeratin 19 Fragment (CYFRA)

\section{Introduction}

The standard treatment of invasive bladder cancer is total cystectomy and lymph node dissection. Recently, neoadjuvant or adjuvant chemotherapy has been seen to improve the prognosis of these patients. Evidence of the utility of neoadjuvant chemotherapy already exists. We can recognize to turn in some cases arter we performed total cystectomy after neoadjuvant chemotherapy for muscle invasive bladder cancer (MIBC) results of T0 can be achieved.

However, since total cystectomy impairs the patient's quality of life, other therapeutic options, including adjuvant radiation therapy or transcatheter arterial infusion (TAI), have been tried. TAI has been found to result in concentration of the anticancer agents in the bladder, making it superior to systemic chemotherapy. We used TAI to administer neoadjuvant chemotherapy in patients with invasive bladder cancer and T1G3 cancer, perform-

"Corresponding author. ing transurethral resection of bladder tumor (TUR-BT) before total cystectomy. Further, we evaluated the possibility of bladder preservation using this treatment method. In prostatic cancer patients, prostate-specific antigen (PSA) is accepted as the standard marker for evaluation of therapy. However, although carbohydrate antigen 19 9 (CA19-9) and squamous cell cancer (SCC) antigen have been suggested as markers for the therapeutic evaluation of invasive bladder cancers, a single accepted marker does not exist. We previously reported that urinary cytokeratin 19 fragment (CYFRA) may be a marker of bladder cancer [1]. In this study, we investigated the usefulness of TAI therapy for invasive bladder cancer and whether serum CYFRA, which is a tumor marker of lung cancer, can be used as a marker of bladder cancer.

\section{Patients and Methods}

This was a case-control study. A total of 56 patients out 
of those with bladder cancer who underwent initial treatment in our department between November 2001 and November 2010 were found to have greater than stage pT1G3 and pT2 cancer on initial TUR-BT. The median age of these patients was 73 years (range, $43-77$ ). The characteristics of the patients are summarized in Table 1. Staging and evaluation of the characteristics of each tumor was done according to the TNM classification and guidelines of the World Health Organization as follows:

1) The tumor was resected by a pure cut to minimize tissue damage by an electric current, such that the entire or part of the tumor and the muscle layer were included in a single piece;

2) Staging for lymph nodes and distant metastasis was performed by computed tomography (CT) and bone scintigraphy.

TAI was performed when the patient accepted the therapy upon our recommendation. Written informed consent was obtained from all the patients. The anticancer agents administered by TAI consisted of Cisplatin (CDDP) $100 \mathrm{mg} \cdot \mathrm{m}^{-2}$, methotrexate (MTX) $30 \mathrm{mg} \cdot \mathrm{m}^{-2}$ and doxorubicin (ADR) $20 \mathrm{mg} \cdot \mathrm{m}^{-2}$ body surface area. The tip of the transarterial catheter was positioned just above the bifurcation of the abdominal aorta. Embolization of the superior gluteal artery bilaterally and application of bilateral thigh tourniquets were routinely performed to prevent necrosis of the gluteal and thigh muscles and to increase the concentration of anticancer agents in the vessels of the pelvis.

Our therapeutic protocol consisted of two courses of TAI after the initial TUR-BT. Following this, a second TUR-BT of the bladder was performed to obtain a biopsy specimen. In case of superficial or undetectable tumors on TUR biopsy, the bladder was preserved, while advanced cases and those with residual bladder tumors

Table 1. Baseline characteristics of the bladder cancer patients.

\begin{tabular}{lcc}
\hline No. of men/women & $46 / 10$ & \\
\hline Median age (years) & $70(43-87)$ & \\
Pathology & $(\mathrm{n})$ & \\
Urothelial carcinoma (UC) & 54 & $96 \%$ \\
UC + Squamous cell carcinoma & 2 & $4 \%$ \\
(SCC) & & \\
Clinical stage & $(\mathrm{n})$ & \\
Tis & 1 & $1.7 \%$ \\
Ta & 0 & $0 \%$ \\
T1 & 15 & $26.7 \%$ \\
T2 & 37 & $66.5 \%$ \\
T3 & 2 & $3.4 \%$ \\
T4 & 1 & $1.7 \%$ \\
Grade & $(\mathrm{n})$ & \\
1 & 0 & $0 \%$ \\
2 & 5 & $8.9 \%$ \\
3 & 51 & $91.1 \%$ \\
Median serum CYFRA $\left(\mathrm{ng} \cdot \mathrm{ml}^{-1}\right)$ & $2.24(0.8-8.1)$ & \\
(range) & & \\
\hline
\end{tabular}

were treated by total cystectomy or systemic chemotherapy.

After TUR-BT, cystoscopy, urinary cytological examination and determination of soluble cytokeratin 19 fragments in serum and urine were performed every 3 months for 2 years, every 6 months from 3 to 5 years, and annually after 5 years. Chest radiographs and pelvic CT were performed every 6 months for 3 years, and annually thereafter. In cases with visible tumors or hyperemic mucosa in the bladder on cystoscopy, positive urinary cytological findings or markedly elevated serum or urine CYFRA 21-1, transurethral biopsy was performed to detect disease recurrence.

Five year overall survival rates and cancer-specific survival rates were calculated using the Kaplan-Meier method. We also determined whether serum CYFRA, which is a tumor marker of lung cancer, can be used as a marker of bladder cancer.

\section{Results}

Pathology studies of the bladder cancers prior to the 2 courses of TAI indicated that the tumors were urothelial carcinomas in 54 patients $(96 \%)$, and mixed squamous cell and urothelial carcinomas in the remaining 2 patients (4\%). The pathological stages in the patients were as follows: Tis in 1 patient, T1 in 15 patients $(26 \%)$, T2 in 37 patients $(66.5 \%)$, and T3 or higher in 3 patients. Five patients $(8.9 \%)$ were Grade 2 and 51 patients $(91.1 \%)$ were Grade 3.

Table 2 shows the results of pathological evaluation of the TUR-BT specimens after 2 courses of TAI. Complete pathological response (CR) was recognized in $63.1 \%$ patients. $15.7 \%$ of the patients revealed non-muscle-invasive cancer. These patients were surgically the CR group after TUR-BT. Non-responders (stable disease: SD or progressive disease: PD) comprised $21 \%$ of the total cases.

Disease-specific survival rates are shown in Table 3. Forty-eight of the 56 patients were still alive during the 4 - 150 months observation period (Figure 1). Eight of the patients succumbed to their disease within 53 - 1779 days

Table 2. Response to therapy.

\begin{tabular}{lc}
\hline Complete Response (CR) & $63.1 \% 24 / 38$ \\
Surgically CR & $15.7 \% 6 / 38$ \\
Stable disease (SD) & $21 \% 8 / 38$ \\
Progressive disease (PD) & $0 \%$ \\
\hline
\end{tabular}

Table 3. Disease-specific survival rates.

\begin{tabular}{cc}
\hline Stage & 5-year survival rate \\
\hline G3, pT1 & $85.7 \%$ \\
T2 & $77.8 \%$ \\
$\geq \mathrm{T} 3$ & $66.7 \%$ \\
\hline
\end{tabular}




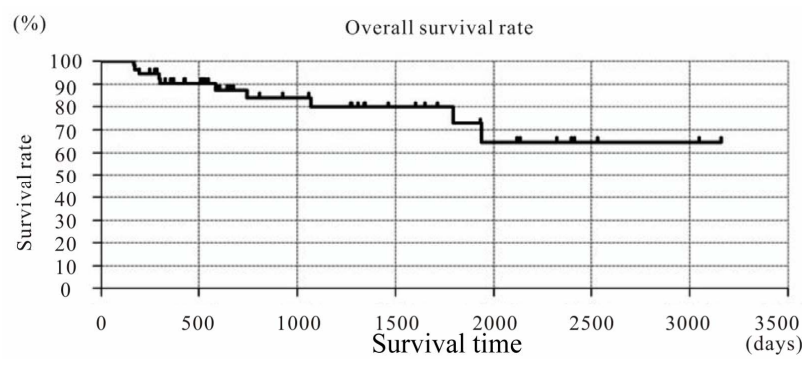

Figure 1. Overall survival rate treated with TAI for muscle invasive bladder cancer by Kaplan-Meier method.

(mean 488.6 days), the cause of death in all of them being local recurrence of bladder cancer or metastasis. None of the patients died due to non-cancer causes. Therefore, disease specific survival and overall survival were similar. The 8 patients who died had been treated with systemic chemotherapy or cystectomy, while some patients died more than 5 years after TAI treatment.

Bladder preservation rates according to disease stage are shown in Table 4. Of the 48 patients still alive, 24 patients were cancer-free (42.8\%) (Figure 2).

The adverse effects of TAI observed in the 56 patients included anemia and vomiting, although none of them were greater than grade 3 in severity. Grade 3 leukopenia was observed in 6 cases $(10.7 \%)$, although it improved with the administration of granulocyte colony-stimulating factor (G-CSF). In addition, Grade 3 thrombocytopenia was observed in 1 case $(1.8 \%)$, for which platelet transfusion was performed.

We examined serum CYFRA levels before and after treatment with TAI, and compared them in terms of bladder preservation and non-preservation. Average serum CYFRA in all cases was $2.24 \mathrm{ng} \cdot \mathrm{ml}^{-1}$ (range, $0.8-8.1$ ). Among the patients who died due to their disease, comparison of the normal CYFRA group $\left(<3.5 \mathrm{ng} \cdot \mathrm{ml}^{-1}\right)$ and the elevated serum CYFRA group $\left(>3.5 \mathrm{ng} \cdot \mathrm{ml}^{-1}\right)$ demonstrated a relative risk of death of 3.51 and odds ratio of 5.37 (Table 5(a)). Bladder outcomes in terms of serum CYFRA levels are shown in Table 5(b). Comparison of bladder preservation and non-preservation in patients with serum CYFRA levels greater than $3.5 \mathrm{ng} \cdot \mathrm{ml}^{-1}$ indicated no significant difference between them, with an odds ratio of 1.4.

\section{Discussion}

TAI has been employed at some facilities for administration of neoadjuvant therapy, along with total cystectomy, in inoperable cases in the elderly or those with complications, and for improving survival in advanced cancer patients. There is some evidence about the efficacy of systemic neoadjuvant chemotherapy with methotrexate, vinblastine, doxorubicin and cisplatin (MVAC), or gemcitabine plus cisplatin (GC) for muscle-invasive bladder
Table 4. Bladder preservation rate according to disease stage.

\begin{tabular}{cc}
\hline Stage & Bladder preservation rate \\
\hline G3, pT1 & $85.7 \%$ \\
T2 & $63.8 \%$ \\
$\geq \mathrm{T} 3$ & $33.3 \%$ \\
\hline
\end{tabular}

Table 5. Patient outcomes in terms of serum CYFRA levels.

(a)

\begin{tabular}{ccccc}
\hline & & Dead & Alive & Total \\
\cline { 2 - 5 } Serum & Over & 3 & 6 & 9 \\
CYFRA & Under & 4 & 43 & 47 \\
3.5 ng.ml-1 & Total & 7 & 49 & 56 \\
& & & \\
\hline
\end{tabular}

(b)

\begin{tabular}{|c|c|c|c|c|}
\hline & \multicolumn{4}{|c|}{ Bladder preservation or cystectomy/death } \\
\hline Serum & & $\begin{array}{c}\text { Total } \\
\text { cystectomy } \\
\text { or death }\end{array}$ & Preservation & Total \\
\hline $\begin{array}{c}\text { CYFRA } \\
3.5\end{array}$ & Over & 3 & 6 & 9 \\
\hline $\mathrm{ng} \cdot \mathrm{ml}^{-1}$ & Under & 12 & 35 & 47 \\
\hline & Total & 15 & 41 & 56 \\
\hline
\end{tabular}

NS (There was no difference in the bladder preservation).

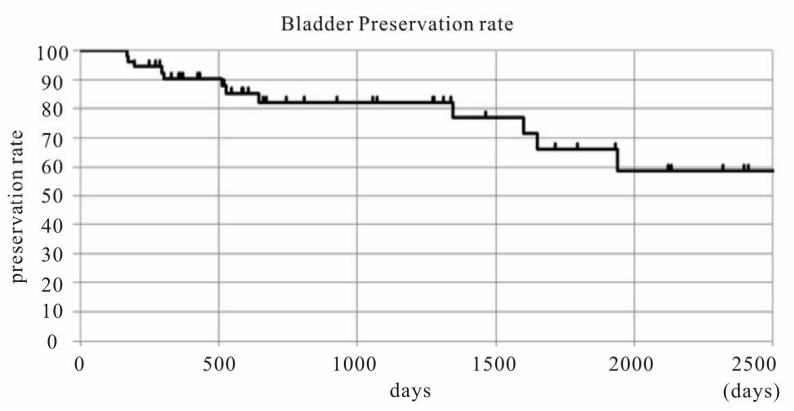

Figure 2. The success rate of bladder preservation treated TAI for muscle invasive bladder cancer by Kaplan-Meier method.

cancer (MIBT), although few studies have reported on neoadjuvant therapy administered by TAI.

In this study, we performed TAI for neoadjuvant chemotherapy against MIBT. We preserved the bladder in some patients who had non-invasive cancer or CR on pathological evaluation of the TUR-BT specimen after chemotherapy. In other studies, the drugs administered by TAI included CDDP + doxorubicin, CDDP + $5 \mathrm{FU}$, and CDDP + Gemcitabine. In our hospital, we typically use CDDP + Doxorubicin + MTX for TAI. The common drug in all these chemotherapeutic regimens is CDDP. Using our drug combination administered by TAI, we found a 5 -year survival rate of $64.5 \%$ by Kaplan-Meier analysis, including the patients who dropped out of the study. In other reports using other drugs, the overall 3- to 5 -year survival rates ranged from $54 \%$ to $72 \%$. Our results are therefore comparable to those of these previous 
studies.

Generally, the 5-year survival rate of invasive bladder cancer (pT2 pT4) is $45 \%-52 \%$ in patients with bladder preservation and $36 \%-49 \%$ in those who undergo complete removal of the bladder. In one study in which the bladder was preserved by using chemotherapy + radiation in 348 cases, the 5 -year survival rate was $61 \%$ in $\mathrm{T} 2$ cases, and $41 \%$ in cT3 $\sim \mathrm{T} 4$ disease [2]. In our study, the 5 -year survival rate was $73.5 \%$ in $\mathrm{T} 2$ cases, indicating that our TAI treatment is not inferior even in comparison with bladder preservation using chemotherapy + radiation [3-5]. With respect to bladder preservation, in our study the bladder preservation rate (probability of survival and preserving the bladder) was $58.1 \%$. Mokarim et al reported a bladder preservation rate following TAI of $70 \%-71 \%$. It is possible that TAI + radiation is a more effective treatment option for MIBT [6,7].

Although NMP22 and BTA have been used as urinary markers of bladder cancers, no serum marker has been established. In this study, we investigated the correlation between the effect of treatment and serum CYFRA levels. CYFRA 21-1 is a cytokeratin 19 fragment that is detected in blood and other body fluids. Serum CYFRA levels are increased by hematogenous metastasis and primary tumor growth of lung cancer, especially squamous cell carcinoma.

CYFRA 21-1 has been reported as a tumor marker for various cancers, such as esophagus and colon cancer [8]. In addition, CYFRA is clinically used for the diagnosis, disease evaluation and follow-up of colon cancer $[9,10]$. Andreadis et al. reported that serum CYFRA levels were elevated in $66 \%$ patients with invasive bladder cancer with metastasis [11]. In our study, patients with high serum levels of CYFRA had a higher relative risk of death of 3.51 (odds ratio, 5.37) compared to patients with normal levels. It has been suggested that serum CYFRA levels can be used as a prognostic factor for urothelial carcinoma. In this study, of the 6 cases with elevated serum CYFRA levels before TAI, 2 cases were fatal. Although serum CYFRA levels after TAI were measured in only 1 of the 2 cases, serum CYFRA remained high even after TAI in this case. On the other hand, serum CYFRA levels returned to normal in the other 4 patients in whom it was elevated before TAI, and these patients also survived their disease. Recurrence of superficial bladder cancer was observed in 2 of the 4 patients who had high levels of CYFRA, although they are now cancer-free as a result of intravesical BCG therapy as add-on therapy. It is suggested that cases with high serum CYFRA are also likely to be positive for lymph node metastasis. On the other hand, serum CYFRA levels returned to normal in patients with CR. Thus, we believe that serum CYFRA levels are useful not only for determining therapeutic effect, but also disease prognosis.

\section{Conclusion}

These results suggest that, although total cystectomy is usually indicated for T1G3 bladder cancers, bladder preservation and control of micrometastases can be achieved by performing TAI after radical TUR-BT. Our results also suggest the utility of monitoring serum CYFRA to assess the response to therapy.

\section{Limitation}

In this study, the number of cases is small. Therefore, it is necessary to increase the number of cases in the future. In addition, the observation period is short in some cases. So, for these cases, it is necessary to observe longer periods in the future.

\section{REFERENCES}

[1] Y. Senga, G. Kimura, T. Hattori and K. Yoshida, "Clinical Evaluation of Soluble Cytokeratin 19 Fragments (CYFRA 21-1) in Serum and Urine of Patients with Bladder Cancer," Urology, Vol. 48, No. 5, 1996, pp. 703-710. doi:10.1016/S0090-4295(96)00253-1

[2] G. Gakis, J. Efstathiou, S. P. Lerner, et al., "ICUD-EAU International Consultation on Bladder Cancer 2012: Radical Cystectomy and Bladder Preservation for Muscle-Invasive Urothelial Carcinoma of the Bladder," European Urology, Vol. 63, No. 1, 2013, pp. 45-57. doi:10.1016/j.eururo.2012.08.009

[3] H. Azuma, K. Yamamoto, T. Inamoto, et al., "Total Cystectomy versus Bladder Preservation Therapy for Locally Invasive Bladder Cancer: Effect of Combined Therapy Using Balloon-Occluded Arterial Infusion of Anticancer Agent and Hemodialysis with Concurrent Radiation," American Journal of Clinical Oncology, Vol. 32, No. 6, 2009, pp. 592-606. doi:10.1097/COC.0b013e318199fb42

[4] H. Azuma, T. Inamoto, K. Takahara, et al., "Neoadjuvant and Adjuvant Chemotherapy for Locally Advanced Bladder Carcinoma: Development of Novel Bladder Preservation Approach, Osaka Medical College Regimen," International Journal of Urology, Vol. 19, No. 1, 2012, pp. 26-38. doi:10.1111/j.1442-2042.2011.02856.x

[5] H. Azuma, T. Inamoto, N. Ibuki, et al., "Novel Bladder Preservation Therapy for Locally Invasive Bladder Cancer: Combined Therapy Using Balloon-Occluded Arterial Infusion of Anticancer Agent and Hemodialysis with Concurrent Radiation," International Journal of Urology, Vol. 37, No. 4, 2010, pp. 773-785. doi:10.3892/ijo 00000727

[6] A. Mokarim, M. Uetani, I. Sakamoto, et al., "Transcatheter Arterial Infusion of Cisplatin and Doxorubicin in Bladder Cancer," Acta Oncologica, Vol. 36, 1997, pp. 175-181. doi: $10.3109 / 02841869709109227$

[7] S. Ozono, S. C. Kim, K. Takashima, et al., "Intra-Arterial Chemotherapy for Invasive Bladder Cancer," Hinyokika Kiyo, Vol. 45, No. 2, 1999, pp. 133-137.

[8] H. J. Yan, R. B. Wang, K. L. Zhu, et al., "Cytokeratin 19 
Fragment Antigen 21-1 as an Independent Predictor for Definitive Chemoradiotherapy Sensitivity in Esophageal Squamous Cell Carcinoma," Chinese Medical Journal (English Edition), Vol. 125, No. 8, 2012, pp. 1410-1415.

[9] Y. N. Fahmueller, D. Nagel, R. T. Hoffmann, et al., "Predictive and Prognostic Value of Circulating Nucleosomes and Serum Biomarkers in Patients with Metastasized Colorectal Cancer Undergoing Selective Internal Radiation Therapy," BMC Cancer, Vol. 4, No. 12, 2012, p. 5.

[10] W. Sidney, P. C. Vincent, S. Melvin and J. Douglas,
"Transcatheter Intraarterial Infusion of Chemotherapy in Advanced Bladder Cancer," Cancer, Vol. 49, 1982, pp. 640-645.

doi:10.1002/1097-0142(19820215)49:4<640::AID-CNCR 2820490406>3.0.CO;2-6

[11] C. Andreadis, S. Touloupidis, G. Galaktidou, et al., "Serum CYFRA 21-1 in Patients with Invasive Bladder Cancer and Its Relevance as a Tumor Marker during Chemotherapy," Journal of Urology, Vol. 174, No. 5, 2005, pp. 1771-1775. doi:10.1097/01.ju.0000176742.53556.25 